\title{
Muon Acceleration: Neutrino Factory and Beyond*
}

\section{S. Alex Bogacz}

Jefferson Lab

12050 Jefferson Avenue, Newport News, VA 23606, USA

E-mail: bogacz@jlab.org

ABSTRACT: We summarize the current state of a concept for muon acceleration aimed at a future Neutrino Factory and extendable to Higgs Factory. The main thrust of these studies was to reduce the overall cost while maintaining performance by exploring the interplay between the complexity of the cooling systems and the acceptance of the accelerator complex. To ensure adequate survival for the short-lived muons, acceleration must occur at high average gradient. The need for large transverse and longitudinal acceptances drives the design of the acceleration system to an initially low RF frequency, e.g., $325 \mathrm{MHz}$, which is then increased to $650 \mathrm{MHz}$ as the transverse size shrinks with increasing energy. High-gradient normal conducting RF cavities at these frequencies require extremely high peak-power RF sources. Hence superconducting RF (SRF) cavities are chosen. We consider an SRF-efficient design based on a multi-pass (4.5) "dogbone" RLA, extendable to multi-pass FFAG-like arcs.

\footnotetext{
* Work has been authored by Jefferson Science Associates, LLC under Contract No. DE-AC0506OR23177 with the U.S. Department of Energy. The U.S. Government retains a nonexclusive, world-wide license to publish or reproduce this manuscript, or allow others to do so, for U.S. Government purposes.
}

The 19th International Workshop on Neutrinos from Accelerators-NUFACT2017

25-30 September, 2017

Uppsala University, Uppsala, Sweden 


\section{Contents}

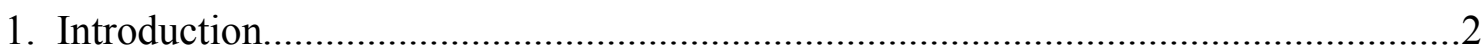

2. Muon Accelerator Complex: Overview..................................................................

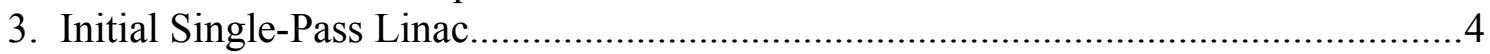

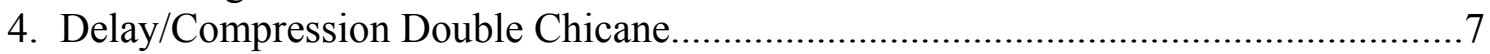

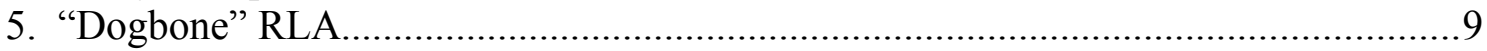

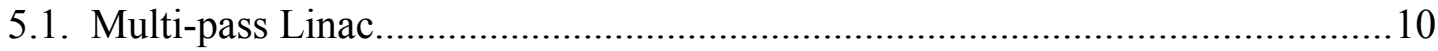

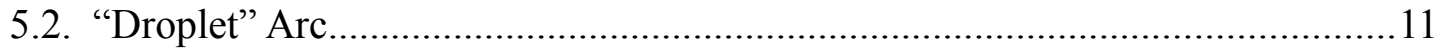

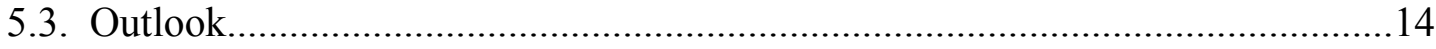

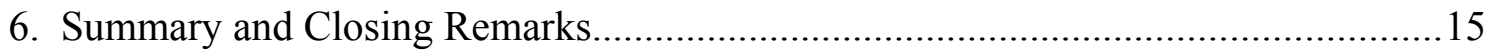

\section{Introduction}

We present key concepts for muon acceleration aimed at a future Neutrino Factory. We follow the leading design options recently identified for NuMAX [1] (5 GeV Neutrino Factory). The main thrust of these studies was to reduce the overall cost while maintaining performance through exploring the interplay between the complexity of the cooling systems and the acceptance of the accelerator complex.

To ensure adequate survival for the short-lived muons, acceleration must occur at high average gradient. The accelerator must also accommodate the phase-space volume occupied by the beam after the cooling channel, which is still large. The need for large transverse and longitudinal acceptances drives the design of the acceleration system to an initially low RF frequency, e.g., $325 \mathrm{MHz}$, which is then increased to $650 \mathrm{MHz}$ as the transverse size shrinks with increasing energy. High-gradient normal conducting RF cavities at these frequencies require extremely high peak-power RF sources. Hence superconducting RF (SRF) cavities are preferred. In the following we choose an SRF gradient of $20 \mathrm{MV} / \mathrm{m}$ at $325 \mathrm{MHz}$ and subsequently $25 \mathrm{MV} / \mathrm{m}$ at $650 \mathrm{MHz}$, which allows survival of about $85 \%$ of the muons as they are accelerated to $5 \mathrm{GeV}$. Significant groundwork, schemes and fundamental building blocks were already laid by the IDS-NF [2] efforts and further refined by MASS [1].

We consider a cost effective scheme for accelerating muon beams based on a multi-pass (4.5) "Dogbone" RLA, extendable to multi-pass FFAG-like arcs. The design is illustrated schematically in Fig. 1. 


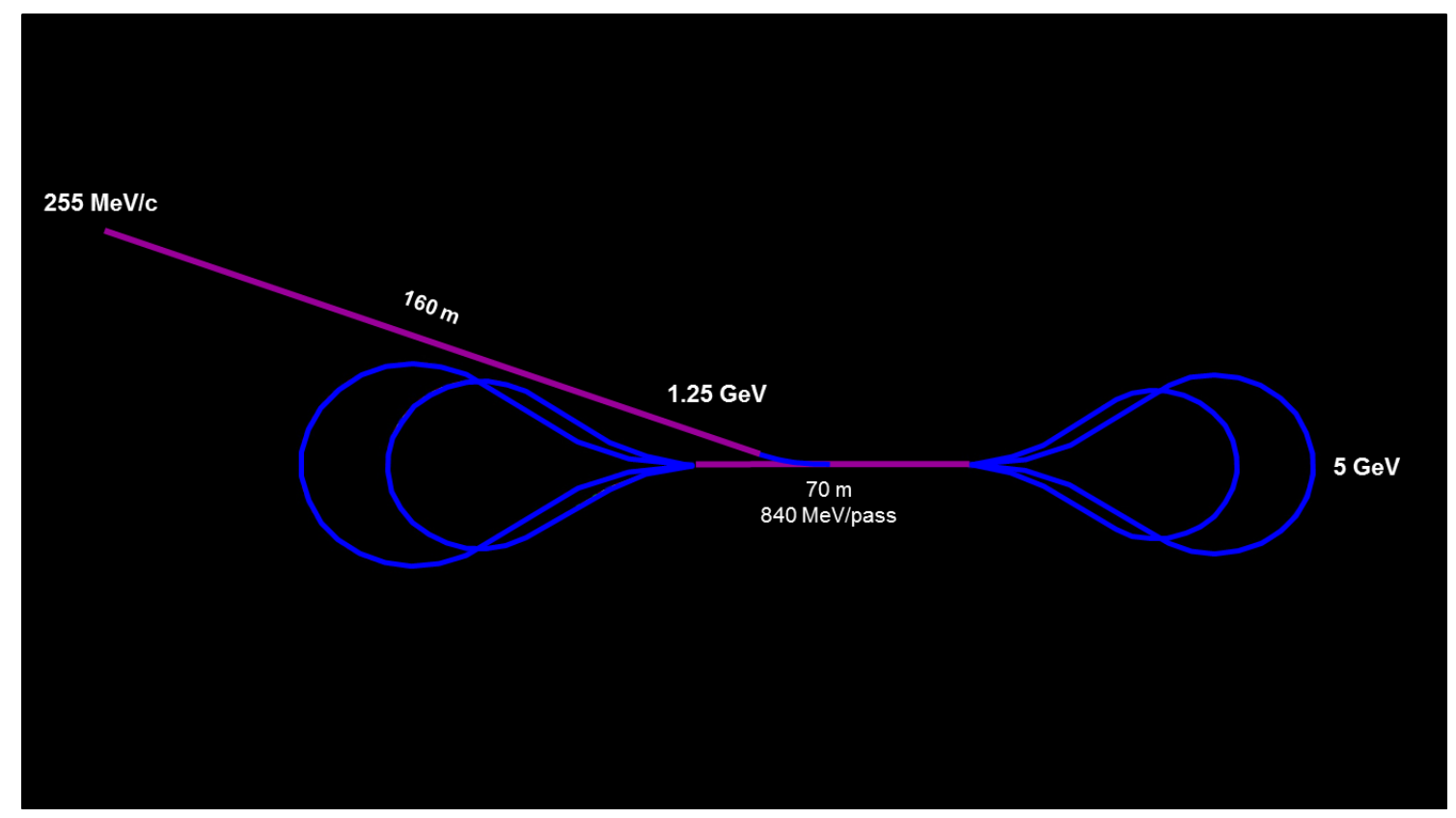

Figure 1. Schematic view of the overall accelerator complex. The droplet arcs supports simultaneous transport of both charge species: at the linac end both $\mu+$ and $\mu$ - travel in the same direction, the first dipole bends them in opposite directions, so they traverse the droplet in clockwise and counter clockwise directions, accordingly. At each consecutive magnet they follow the same trajectory, since they have opposite velocities and charges (same Lorentz force).

\section{Muon Accelerator Complex: Overview}

The proposed muon accelerator complex consists of a single-pass, superconducting linac with $325 \mathrm{MHz}$ RF cavities, accelerating muons to $1.25 \mathrm{GeV}$, that captures the large muon phasespace coming from the bunch rotator (in the case of NuMAX), or the factor-of-2 smaller phasespace after the cooling channel (in the case of the full-luminosity Neutrino Factory). The large acceptance of the linac requires large apertures and tight focusing. This, combined with moderate beam energies, favors solenoid rather than quadrupole focusing for the entire linac [3].

The use of counter-wound solenoids is dictated by a close proximity of multi Tesla solenoid magnets and superconducting cavities. To achieve fast field drop from solenoid to cavity the solenoid has an outer counter-coil, which intercepts its magnetic flux, and the cavity has a SC shielding at its outer surface. That allows one to achieve magnetic field less than $0.1 \mathrm{G}$ inside the cavity. The initial single-pass linac accelerates muons to sufficiently relativistic energy, $1.25 \mathrm{GeV}$, beyond which acceleration using a more efficient and compact higher frequency, $650 \mathrm{MHz}$, linac structure becomes feasible.

Neutrino experiments in $\mathrm{CP}$ violation sector require studies of neutrino and anti-neutrino beams with well understood kinematics. Therefore, proposed accelerator complex is designed to support acceleration of $\mu+$ and $\mu$ - in a fixed field transport system. For simultaneous acceleration of both muon charge species, the transition to the $650 \mathrm{MHz}$ linac requires a halfwavelength path-length delay for one of the muon species. This is facilitated by a path-length delay double chicane, located at the junction between $325 \mathrm{MHz}$ and $650 \mathrm{MHz}$ style linac sections as illustrated in Figure 8. In the chicane muons of different charges follow alternative chicane legs, different in length by a half-wavelength at $650 \mathrm{MHz}$, thus $230.6 \mathrm{~mm}$. 
Then for further acceleration (from $1.25 \mathrm{GeV}$ to $5 \mathrm{GeV}$ ), the linac is followed by a 4.5pass, recirculating linear accelerator (RLA) in a "dogbone" configuration, where acceleration continues using a more compact and efficient $650 \mathrm{MHz}$ SRF structure, while adiabatically decreasing the phase-space volume. The two alternative concepts are described in detail in the following sections.

\section{Initial Single-Pass Linac}

A single-pass linac starting at $255 \mathrm{MeV} / \mathrm{c}$ (276 MeV total energy) raises the total energy of the muons to $1.25 \mathrm{GeV}$. This makes the muons sufficiently relativistic to facilitate further acceleration in the RLA or in the dual-use linac. The initial phase-space of the beam, as delivered by the muon front-end, is characterized by a quite large energy spread; the linac has been designed so that it first confines the muon bunches in longitudinal phase-space, then adiabatically superimposes acceleration over the confinement motion, and finally boosts the confined bunches to $1.25 \mathrm{GeV}$. To achieve a manageable beam-size in the front-end of the linac, short focusing cells (with one 2-cell cavity) are used for the first 22 cryomodules. The beam size is adiabatically damped with acceleration, allowing the short cryomodules to be replaced with 30 intermediate length cryomodules (with one 4-cell cavity). Consequently, the linac is split into two consecutive sections (referred to as the short- and middle-cell linac sections), each section being built of a particular type of cryomodule as shown in Figure 2 [4]. Each linac section is configured with periodic FOFO cells, matched at the section junctions, as illustrated in Figure 2. Periodicity within each section is maintained by scaling the solenoid fields in consecutive cryomodules linearly with increasing momentum [5]. The cavity iris radius limits the physical aperture of the linac. The radius of $15 \mathrm{~cm}$, matched with a 2.5 sigma beam envelope, defines the transverse acceptance (normalized) of the linac as $20 \mathrm{~mm} \cdot \mathrm{rad}$, as shown in Figure 2 (bottom). The 'tight' aperture of 2.5 sigma is validated by special collimation system, which is being considered [4], [5]. The main parameters of the muon linac are summarized in Table 1.

\begin{tabular}{|l|l|}
\hline Injection momentum & $255 \mathrm{MeV} / \mathrm{c}$ \\
\hline Initial normalized acceptance & $20 \mathrm{~mm} \cdot \mathrm{rad}$ \\
\hline Initial longitudinal acceptance, $\Delta \mathrm{pL} / \mathrm{m}_{\mu}$ & $150 \mathrm{~mm}$ \\
momentum spread, $\Delta \mathrm{p} / \mathrm{p}$ & \pm 0.28 \\
bunch length, $\mathrm{L}_{\mathrm{b}}$ & $\pm 252 \mathrm{~mm}$ \\
\hline Number of bunches per pulse & 67 \\
\hline Number of particles per bunch/per pulse & $4.4 \cdot 10^{10} / 3 \cdot 10^{12}$ \\
\hline Bunch frequency/accelerating frequency & $325 / 325 \mathrm{MHz}$ \\
\hline Average repetition rate & $15 \mathrm{~Hz}$ \\
\hline Time structure of muon beam & $6 \mathrm{pulses}$ at $50 \mathrm{~Hz}$ with \\
& $2.5 \mathrm{~Hz}$ repetition rate \\
\hline Average beam power & $120 \mathrm{~kW}$ \\
\hline
\end{tabular}

Table 1. Main Parameters of the Muon Accelerator Driver 


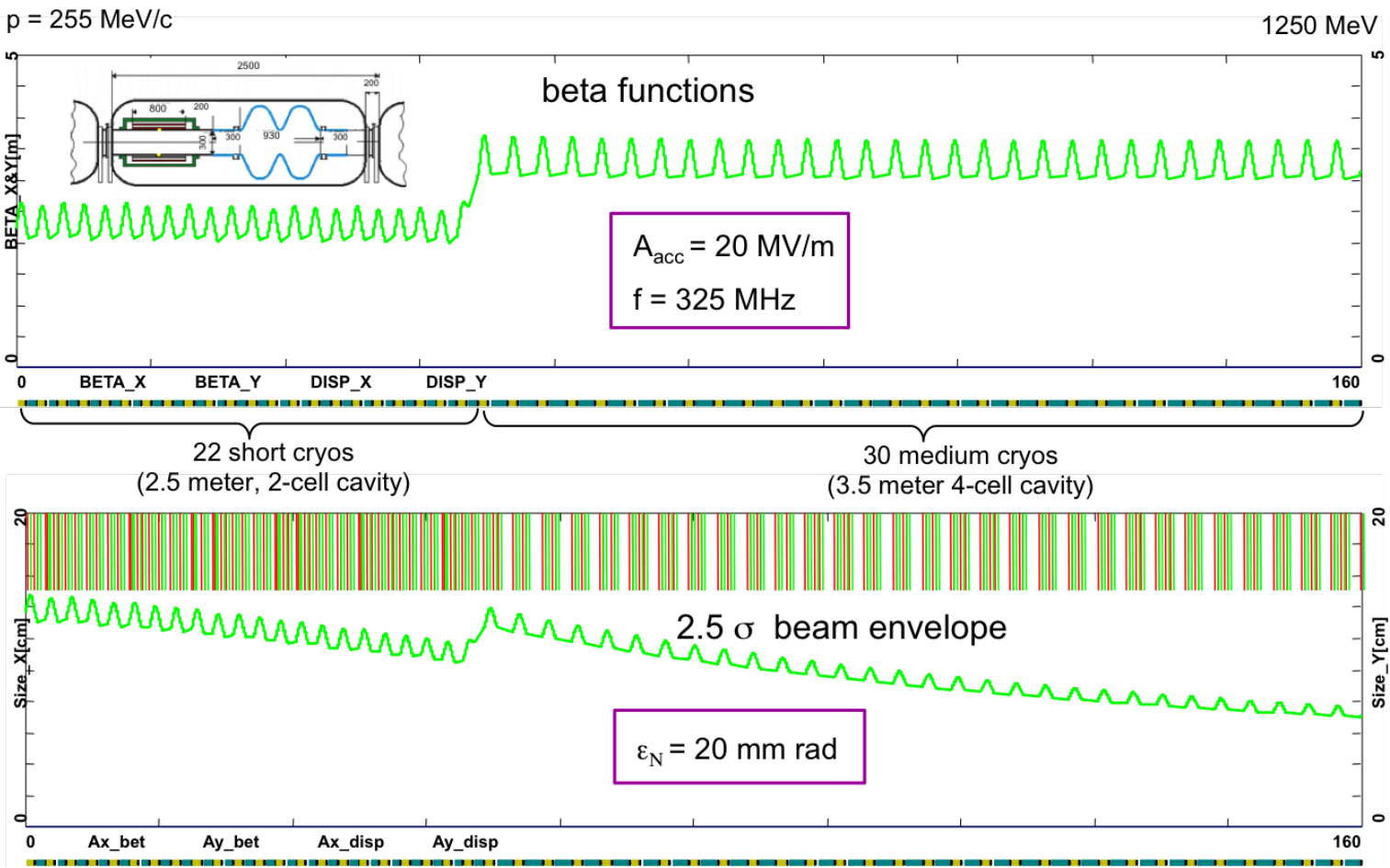

Figure 2. Transverse FOFO optics (top) and the beam envelope of the initial linac; the shortand middle-cell periodic sections are uniformly matched across the junction. The physical aperture radius of $15 \mathrm{~cm}$ (indicated by the vertical bars) defines the transverse acceptance (normalized, rms) as $20 \mathrm{~mm} \cdot \mathrm{rad}$ (bottom).

One of the main requirements of the initial single-pass linac is to compress adiabatically the longitudinal phase-space area in the course of acceleration. The initial longitudinal acceptance of the linac (chosen to be $2.5 \sigma$ ) calls for "full bucket" acceleration, with an initial momentum acceptance $\Delta \mathrm{p} / \mathrm{p}= \pm 28 \%$ and bunch length $\Delta \varphi= \pm 97.5^{\circ}$ (in RF phase). To perform adiabatic bunching one needs to drive rather strong synchrotron motion along the linac [6]. The profile of the RF-cavity phases is organized so that the phase of the first cavity is shifted by $84^{\circ}$ (off crest) and then the cavity phase is gradually changed to about $10^{\circ}$ (off crest) by the end of the linac, as shown in Figure 3a. 
a)

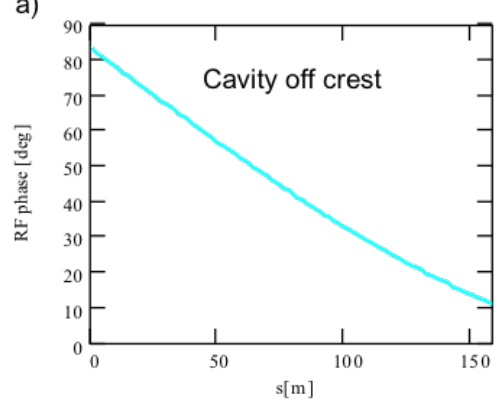

c)

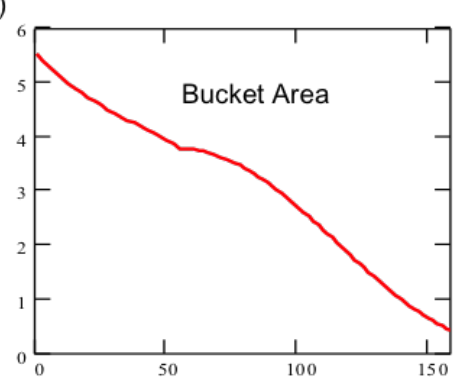

b)

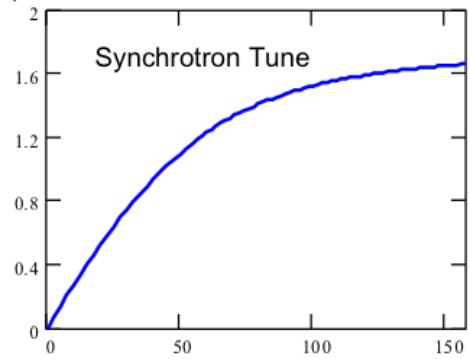

d)

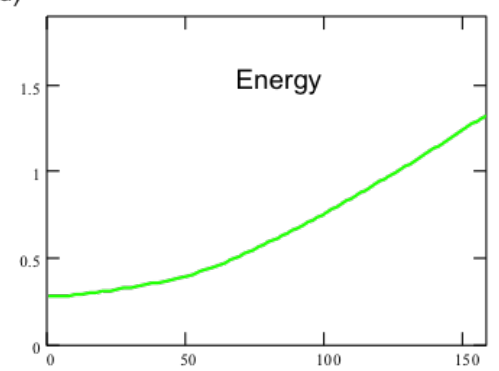

Figure 3. Longitudinal matching of the initial single-pass linac: a) cavity phasing starts far offcrest to capture the beam at low energy, then it moves closer to the crest as the longitudinal emittance decreases with increasing energy; b) the synchrotron phase advances by over one and a half periods from the beginning to the end of the linac; c) the bucket area is initially very large to capture a maximum longitudinal acceptance of $150 \mathrm{~mm}$; it decreases as the bunch is compressed moving closer to crest; d) the rate of energy gain increases as the bunch moves closer to crest.

In the initial part of the linac, when the beam is still not relativistic, the far off-crest acceleration induces rapid synchrotron motion (one and a half full periods along the linac, as shown in Figure 3b), which allows bunch "head" and "tail" to switch places within the RF bucket three times during the course of acceleration. This process [6] is essential for averaging energy spread within the bunch, which ultimately yields desired bunch compression in both bunch-length and momentum spread, as illustrated in Figure 4. To maximize the longitudinal acceptance, the initial position of the bunch is shifted relative to the center of the bucket, to keep the beam boundary inside the separatrix, as illustrated in Figure 4a. The synchrotron motion also suppresses the sag in acceleration [4] for the bunch head and tail as seen in Figure 4b.

Solenoid non-linearities for off axis particles were included in our tracking simulation via OptiM (up to the 3-rd order. Such non-linearity can cause strong non-linear resonance even for small number of lattice periods. Here we studied (via particle tracking) beam emittance as a function of different phase advances per cell after passing a channel with 50 solenoidal lenses. We observed very strong effect of the $1 / 4$ resonance, which spreads in the tune range of [0.21 $0.24]$. The $1 / 6$ resonance is also well visible but does not produce so harmful effect. In practice, it is much smaller because of adiabatic damping of the beam size with acceleration. Taking all that into account we choose tune to be equal to 0.175 , which translates to the phase advance per cell of $63^{\circ}$. The resulting longitudinal phase-space is illustrated in Figure $5 \mathrm{~b}$. 

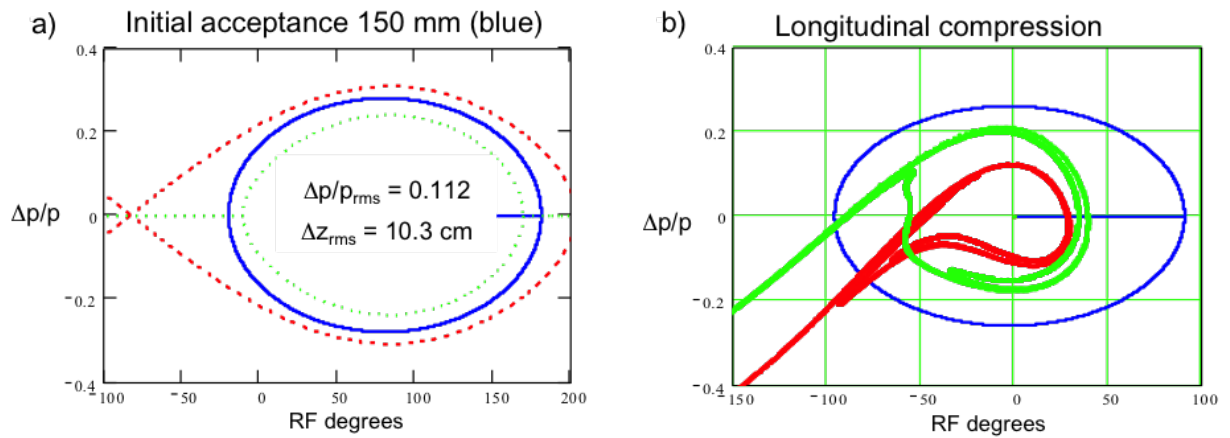

Figure 4. a) Longitudinal acceptance of $150 \mathrm{~mm}$ matched inside the separatrix and optimized for "full bucket" acceleration; b) longitudinal compression in the course of acceleration: initial acceptance (blue) propagated through the first part of the linac (green) and finally fully compressed bunch at the end of the linac (red).

\section{Delay/Compression Double Chicane}

To maintain simultaneous acceleration of both muon charge species, the transition from the $325 \mathrm{MHz}$ to the $650 \mathrm{MHz}$ linac requires a half-wavelength path-length delay for one of the muon species in order to re-establish synchronous acceleration at the new RF frequency, as depicted schematically in Figure 5a. Furthermore, transition to higher RF frequency (650 MHz) introduces an abrupt change in the bucket shape; the new bucket is about a factor of 2 shorter and slightly higher, since larger RF gradient is achievable $(25 \mathrm{MV} / \mathrm{m}$ at $650 \mathrm{MHz}$ vs. $20 \mathrm{MV} / \mathrm{m}$ at $325 \mathrm{MHz}$ ), as illustrated in Figure 5b.

a)

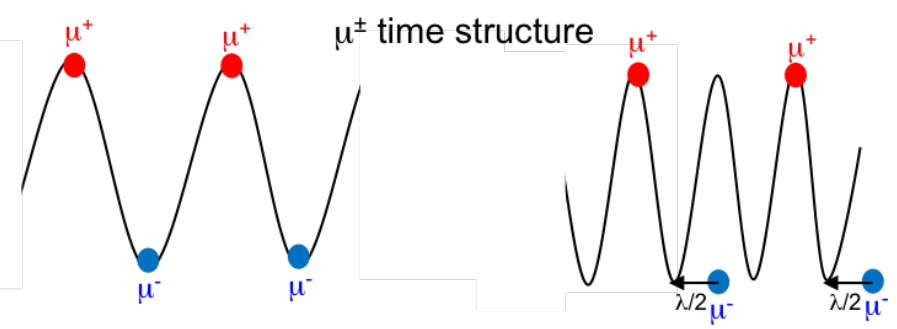

$325 \mathrm{MHz}$ linac
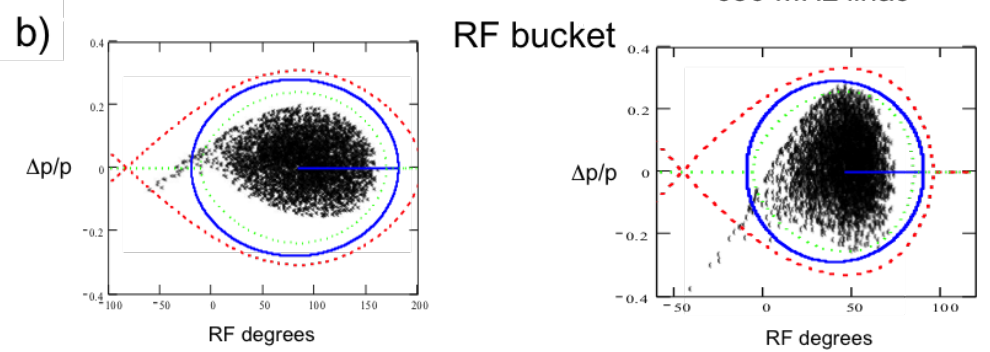

Figure 5. a) Time structure of muon bunches; doubling the RF frequency requires a path-length delay of $\lambda / 2$ for $\mu^{-}$to put them into accelerating buckets of the new frequency; b) change of the bucket length (a factor of 2 shorter when the RF frequency is doubled) requires compression of the bunch-length by about factor of 2 in order to fit the bunch into the new bucket. 
Both effects are addressed by a dedicated path-length delay/compression double chicane, configured with six $30^{\circ}$ bends, where muons of different charges follow alternative chicane legs, different in length by the half-wavelength at $650 \mathrm{MHz}$, as illustrated in Figure 6.
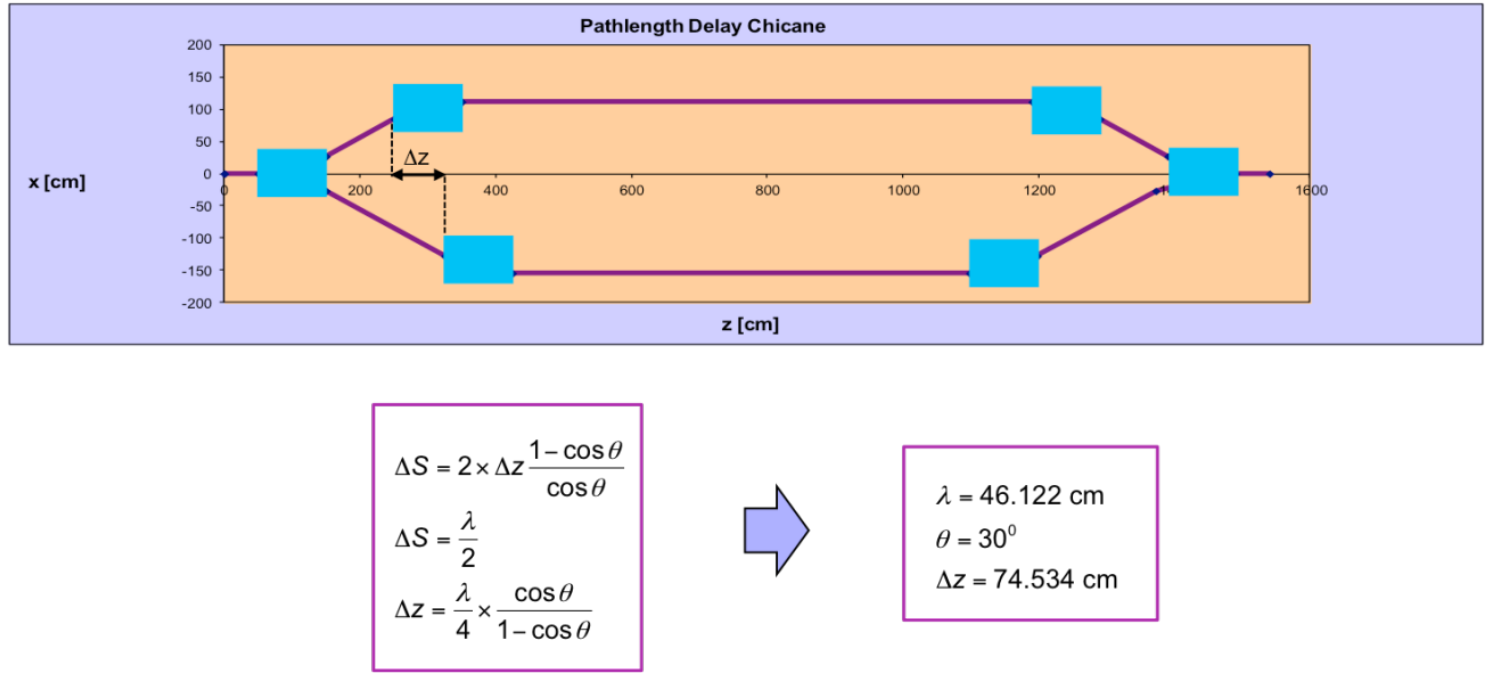

Figure 6. Layout of the path-length delay double chicane, in which muons of different charges follow alternative chicane legs, different in length by a half-wavelength at $650 \mathrm{MHz}$.
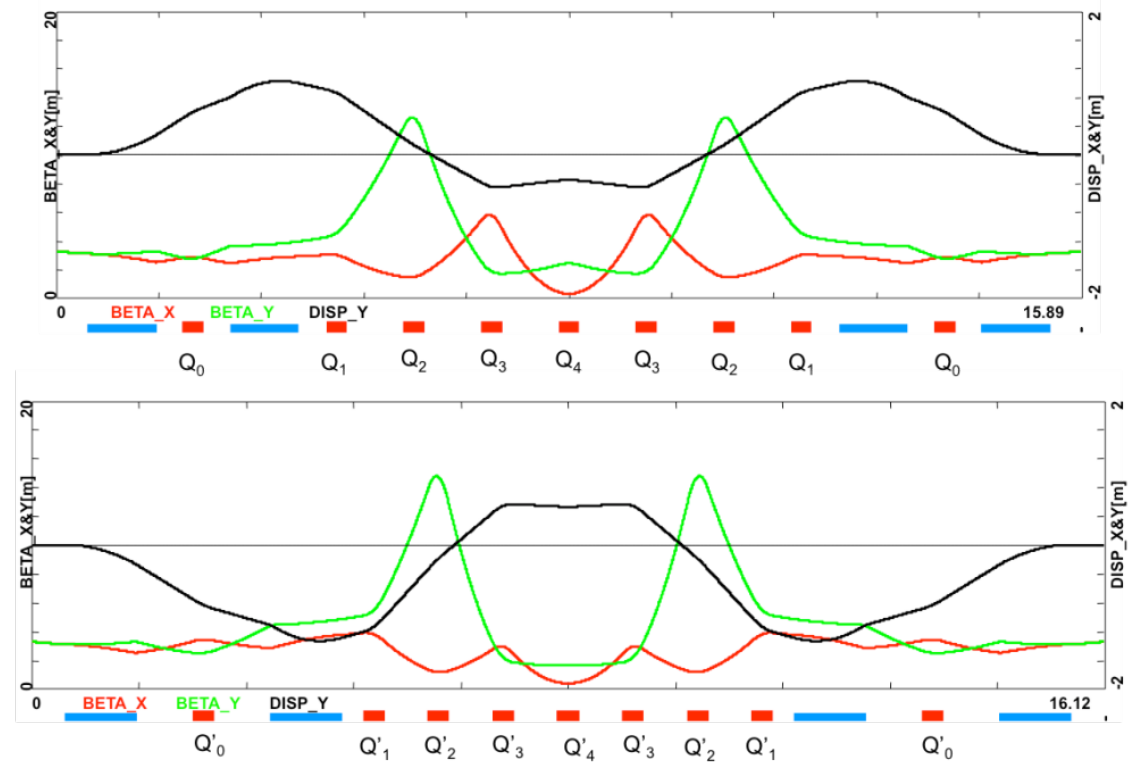

Figure 7. Optics for the two "legs" of the double chicane (top/bottom), featuring mirrorsymmetric vertical achromats, with significant momentum compaction, $\mathrm{M}_{56}=-1 \mathrm{~m}$, matched to the same Twiss functions at the ends.

Each leg of the chicane is configured with 9 quadrupoles powered in mirror-symmetric fashion to provide 5 independent parameters required for tuning of the two betas and two alphas and to suppress the vertical dispersion $(2+2+1)$, as illustrated in Figure 7. Furthermore, this style of optics features large negative momentum compaction, which, combined with an energy 
chirp in the upstream linac, works as an effective quarter-wave rotator in longitudinal phasespace, yielding the bunch-length reduction required for acceleration in the $650 \mathrm{MHz}$ linac, downstream of the chicane. The dynamics of the above process was studied numerically using a particle tracking simulation via the matrix-based code OptiM [9], as illustrated in Figure 8. In the simulation we have assumed a particle distribution that is Gaussian in $6 \mathrm{D}$ phase space with the tails of the distribution truncated at 2.5 sigma, which corresponds to the beam acceptance. Now the muon beam is ready for further acceleration in a $650 \mathrm{MHz}$ linac structure; either in a single-pass "dual-use" linac (Scheme II), or in a multi-pass "dogbone" RLA (Scheme I). Both schemes are described in detail in the following sections.

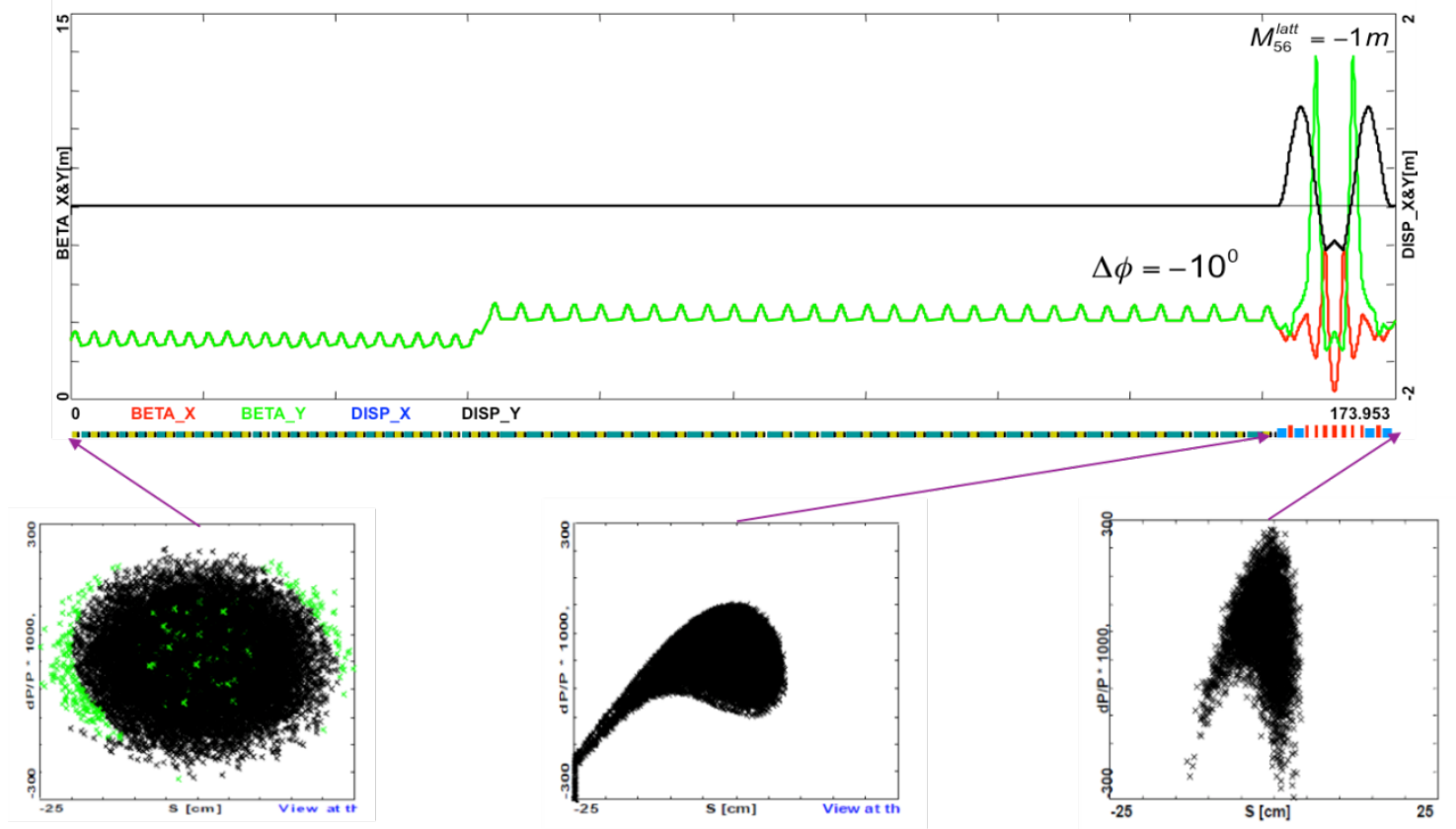

Figure 8. Snapshot of the initial longitudinal phase-space, corresponding to full longitudinal acceptance of $150 \mathrm{~mm}$ (left), transported through the single-pass linac, generating energy chirp by being phased off-crest (middle snapshot), and finally compressed bunch (factor of two shorter) through energy correlated momentum compaction across the chicane (right snapshot).

\section{5. "Dogbone" RLA}

The main virtue of the multi-pass RLA option is its very efficient use of an expensive SRF linac. The "dogbone" RLA is designed to accelerate simultaneously the $\mu^{+}$and $\mu^{-}$beams from $1.25 \mathrm{GeV}$ to $5 \mathrm{GeV}$ to further compress and shape the longitudinal and transverse phase-space [6]. The beam is injected from the single-pass linac via the double chicane (as discussed in Section 3). The injection point into the "dogbone" RLA coincides with the middle of the multipass linac to minimize the effect of phase slippage for the initially $1.25 \mathrm{GeV}$ muon beam accelerated in linacs that are phased for speed-of-light particles. At the ends of the RLA linacs the beams need to be directed into the appropriate energy-dependent (pass-dependent) "droplet" arc for recirculation [7]. The above configuration has already been introduced in Figure 1 (top). The $650 \mathrm{MHz}$ SRF linac is configured with small aperture ( $7.5 \mathrm{~cm}$ radius), higher gradient $(25$ $\mathrm{MV} / \mathrm{m})$ cavities. 


\subsection{Multi-pass Linac}

The injection energy into the RLA and the energy gain per RLA linac $(840 \mathrm{MeV})$ were chosen so that a tolerable level of RF phase slippage along the linac could be maintained $\left(\sim 20^{\circ}\right.$ in RF phase). Earlier studies, [3], [4] showed that injection at the middle of the linac allows one to minimize the phase slippage and maintain balanced transverse optics along the linac (see Figure 11). To suppress chromatic effects, $90^{\circ}$ FODO optics is used as a building block for both the linac and the return arcs [5]. In the linac, the 4-meter-long FODO cells are configured with 4-cell SRF cavities between each of the quadrupoles, except that there are no cavities between the central D magnet and its adjacent $\mathrm{F}$ quadrupoles. The layout and optics of the linac periodic FODO structure are shown in Figure 11a. The injection chicane magnets are located at the middle of the linac (marked in blue).

a) 'half pass' , 1250-1625 MeV

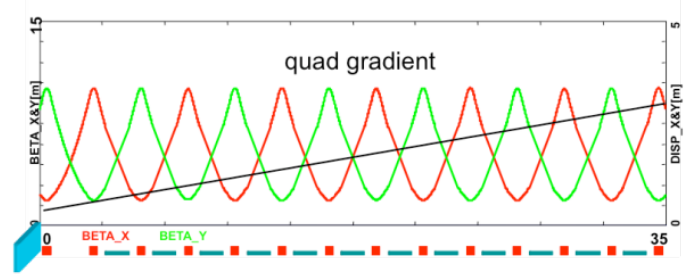

b) 1-pass, $1625-2475 \mathrm{MeV}$

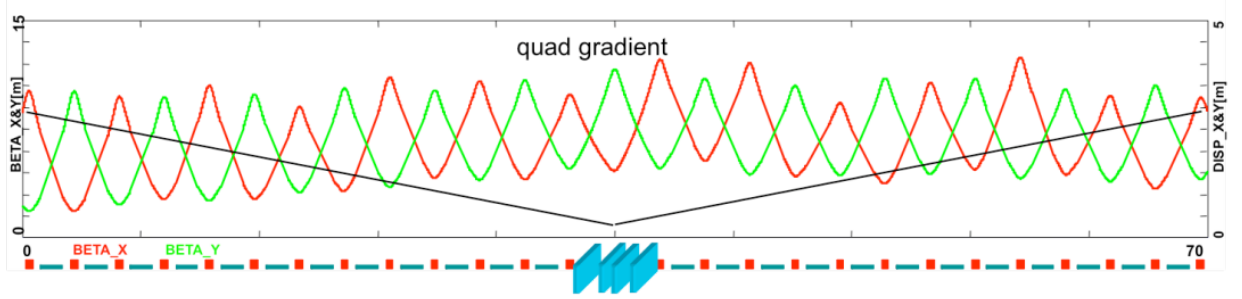

Figure 11. "Bisected" linac optics: the quadrupole gradients are scaled up with momentum for the first half of the linac, then they are mirror reflected in the second half: (a) periodic FODO structure set for the lowest energy "half-pass" through the linac; (b) linac optics for the first "full pass"; underfocusing effects in the first half of the linac are mitigated by reversing the focusing profile in the second half. The blue squares represent re-injection chicane magnets required to close initial orbit bump for higher passes through the linac.

The focusing profile along the linac was chosen so that beams with a large energy spread could be transported within the given aperture. Since the beam is traversing the linac in both directions, a "bisected" focusing profile was chosen for the multi-pass linac [7]. Here, the quadrupole gradients scale up with momentum to maintain $90^{\circ}$ phase advance per cell for the first half of the linac (see Figure 11a), then are mirror reflected in the second half, as illustrated in Figure 11b. Performance of the bisected linac optics for all 4.5 passes is illustrated in Figure 12. 


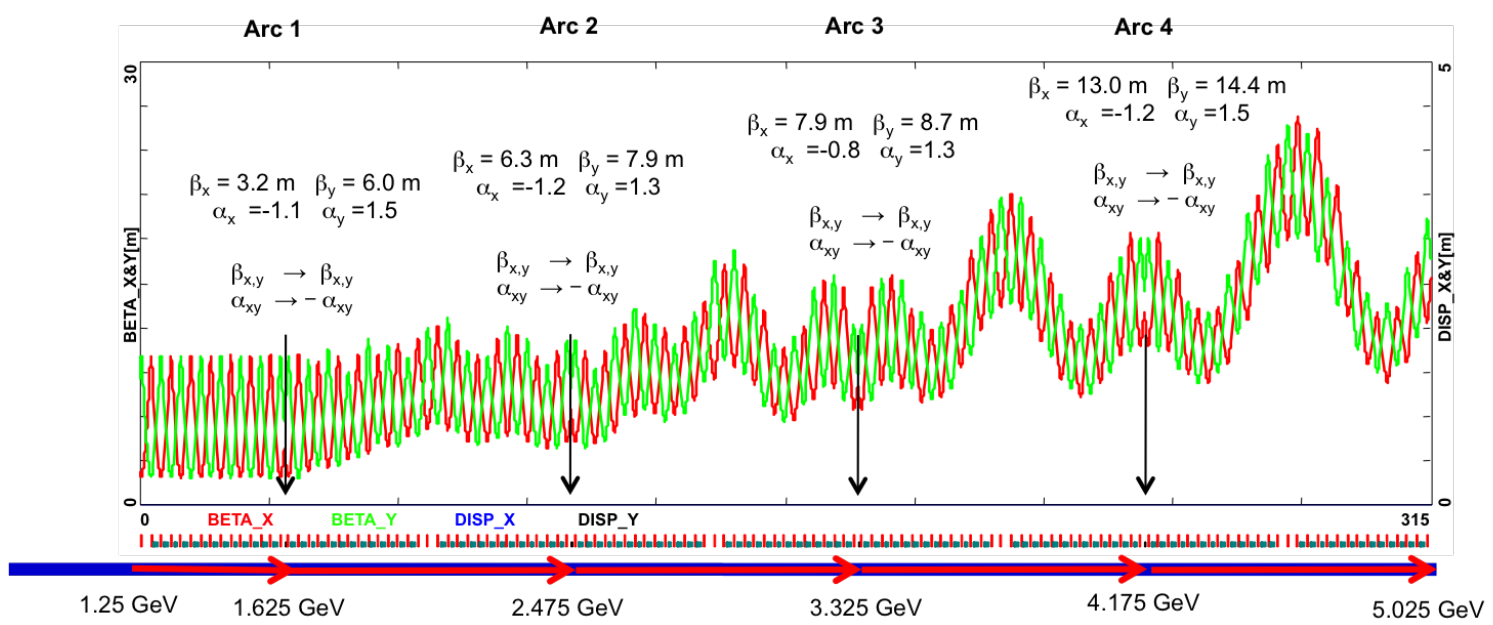

Figure 12. "Bisected" linac optics for all passes, with mirror symmetric arcs inserted as point matrices (arrows). The virtue of the optics is the appearance of distinct nodes in the beta beatwave at the ends of each pass (where the arcs begin), which limits the growth of initial betas at the beginning of each subsequent droplet arc (Arc 1-4), hence eases linac-to-arc matching.

\section{2. "Droplet" Arc}

At the ends of the RLA linac, the beams need to be directed into the appropriate energydependent (pass-dependent) droplet arc for recirculation. The entire droplet-arc architecture [8] is based on $90^{\circ}$ phase-advance cells with periodic beta functions, as depicted in Figure 13. For practical reasons, horizontal rather than vertical beam separation has been chosen. Rather than suppressing the horizontal dispersion created by the spreader, it has been matched to that of the outward arc. This is partially accomplished by removing one dipole (the one furthest from the spreader) from each of the two cells following the spreader. To switch from outward to inward bending, three transition cells are used, from which the four central dipoles are removed. The two remaining dipoles at the ends bend in the same direction as the dipoles to which they are closest. The transition region, across which the horizontal dispersion switches sign, is therefore composed of two such cells. To facilitate simultaneous acceleration of both $\mu^{+}$and $\mu^{-}$bunches; mirror symmetry is imposed on the droplet arc optics (oppositely charged bunches move in opposite directions through the arcs). This puts a constraint on the exit/entrance Twiss functions for two consecutive linac passes, namely: $\beta_{n \text { out }}=\beta_{n+1}$ in and $\alpha_{n \text { out }}=-\alpha_{n+1}$ in, where $n=0,1,2 \ldots$ is the pass index. The complete droplet arc optics for the lowest-energy pair of arcs is shown in Figure 13. 

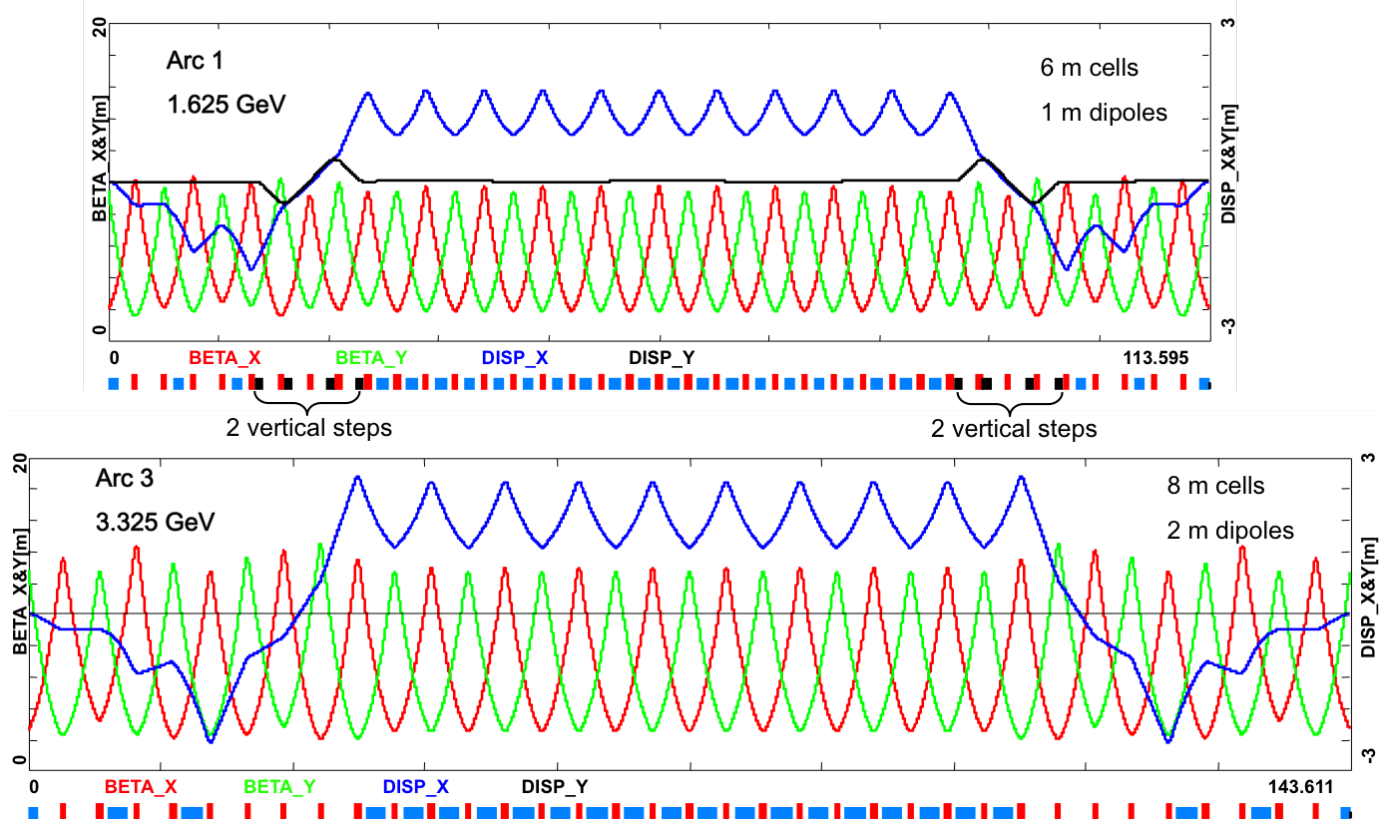

Figure 13. Droplet arc optics for a pair of arcs on one side of the "dogbone," Arc 1 and Arc 3.

All higher arcs are based on the same principle as Arc 1, with gradually increasing cell length (and dipole magnet length) to match naturally to the increasing beta functions dictated by the multi-pass linac. The quadrupole strengths in the higher arcs are scaled up linearly with momentum to preserve the $90^{\circ}$ FODO lattice. The physical layout of the above pair of droplet arcs is illustrated in Figure 14. The momentum compaction in each droplet arc, $\mathrm{M}_{56}=6.5 \mathrm{~m}$, was optimized (via longitudinal phase-space simulation) to provide adequate longitudinal compression for energy chirp in the linac accomplished by setting the linac $5 \mathrm{deg}$. off crest. This effect, yields further compression of the longitudinal phase-space as the beam is accelerated. 


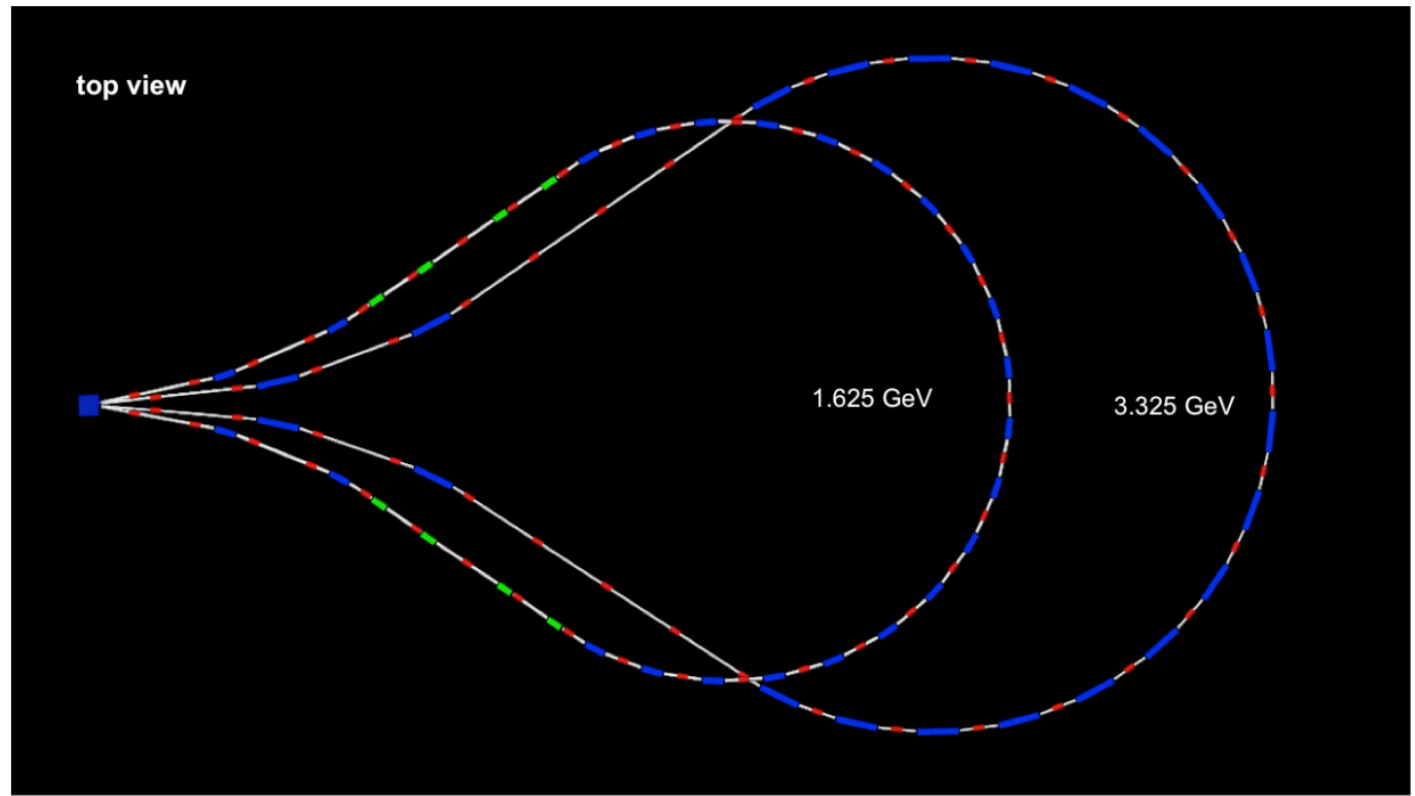

side view

$1.625 \mathrm{GeV}$

$3.325 \mathrm{GeV}$

Figure 14. Layout of a pair of arcs on one side of the "dogbone" RLA: Arc 1 and Arc 3, top and side views, showing vertical two-step "lift" of the middle part of Arc 1 to avoid interference with Arc 3

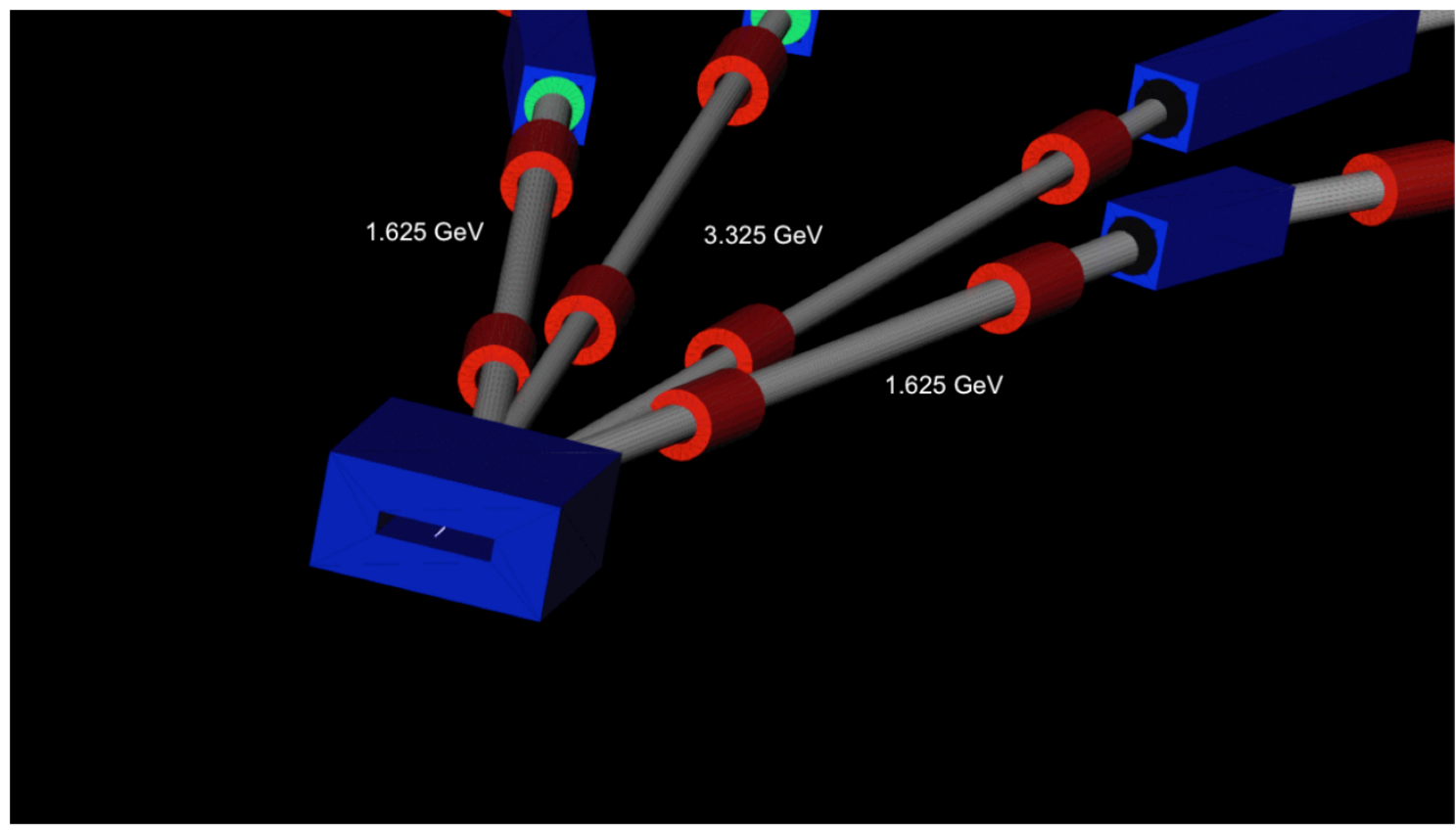

Figure 15. Switchyard layout, featuring a common dipole (blue), separating beams horizontally and directing them to the corresponding arcs. Initial quadrupoles (red) define Spreader/Recombiner optics. 
The muon beam intensity is sufficiently high (see Table 1) therefore the beam loading has to be taken into account. It causes the RF voltage droop by $\sim 0.5 \%$ per pass leading to $\sim 2.3 \%$ loss in acceleration for the bunch tail on the last pass. It is comparable with energy aperture of the highest arc, therefore, additional optics tuning is needed to account for resulting energy droop. Another effect is that the first and the last bunch see different accelerating voltage and experience different longitudinal dynamics. Fortunately, accelerating off-crest resolves this problem as well. In this case, after acceleration in the first pass through the linac, the last bunch experiences less acceleration; but then because of smaller energy the bunch comes faster through the first arc and it is accelerated with smaller RF phase causing higher acceleration in the subsequent linac pass. In summary, the bunch center of the last bunch experiences synchrotron motion relative to the center of the first bunch.

\subsection{Outlook}

In summary, a "dogbone" RLA is a fast, compact and efficient way of accelerating muon beams to medium and high energies by reusing the same linac for multiple passes, where the different energy passes coming out of the linac are separated and directed into individual (single energy) return arcs for recirculation. However, each pass through the linac requires a separate fixed energy arc, hence increasing the complexity, size, and cost of the RLA.

As an alternative, we have also recently proposed a novel return arc optics design based on combined function magnets with variable dipole and quadrupole field components. This allows two consecutive passes with very different energies to be transported through the same string of magnets [10-13].

Each droplet arc consists of a $60^{\circ}$ outward bend, a $300^{\circ}$ inward bend and another $60^{\circ}$ outward bend so that the net bend is $180^{\circ}$. This arc geometry has the advantage that if the outward and inward bends are composed of similar cells, the geometry automatically closes without the need for any additional straight sections, making it simpler and more compact. The super cells constituting the arc are designed to satisfy the following basic conditions:

- Each super cell possesses periodic solutions for the orbit and the Twiss functions

- At the beginning and at the end of each super cell, the periodic orbit offset, dispersion and their slopes are all zero.

These conditions are met at both momenta. The former condition ensures that the super cells bending in the same direction are optically matched while the latter one ensures optical matching of the cells bending in the opposite directions. The latter condition also implies that the beam is centered in the linac and that the linac is dispersion free.

A proof-of-principle droplet arc composed of identical $60^{\circ}$ bending cells - two outwardand five inward-bending cells - has been presented [11]. Such a design provides a greater compactness than, for instance, an FFAG lattice with its regular alternating bends and is expected to possess a large dynamic aperture characteristic of linear-field lattices. A basic building block of this proposed optics scheme is illustrated in Figure 16, which shows solutions for the periodic orbit, dispersion, and beta functions of the outward-bending supercell at 1.2 and $2.4 \mathrm{GeV} / \mathrm{c}$, respectively. 
a)
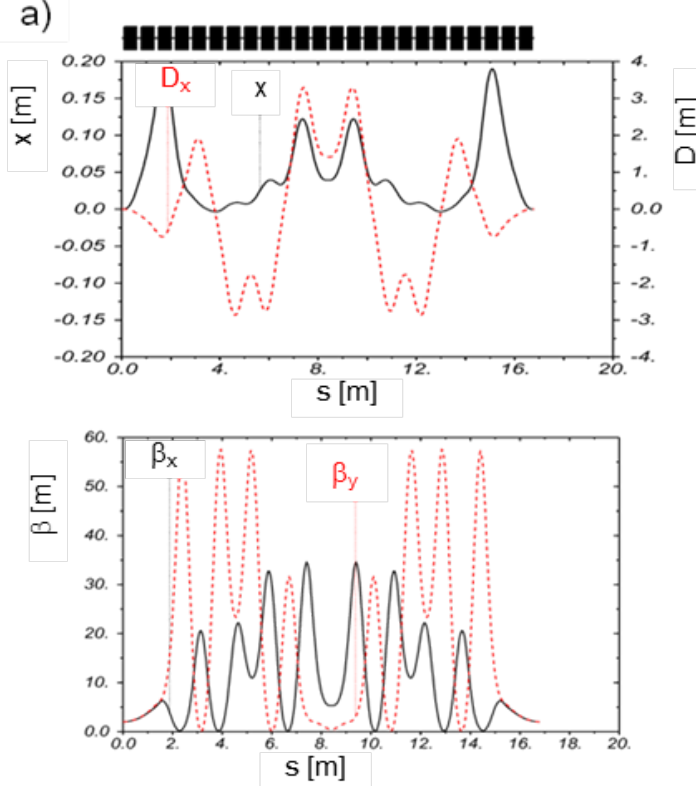

b)
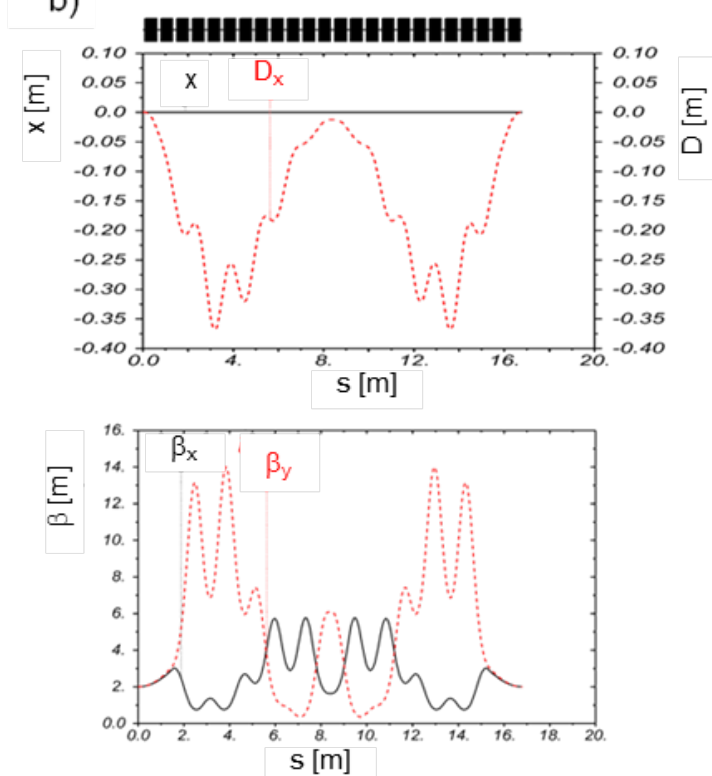

Figure 16. Periodic orbits, dispersion (top) and beta functions (bottom) of a) $1.2 \mathrm{GeV} / \mathrm{c}$ and b) $2.4 \mathrm{GeV} / \mathrm{c}$ outward bending supercells.

Such a solution [14] combines compactness of design with all the advantages of a linear NS-FFAG [13], namely, large dynamic aperture and momentum acceptance essential for largeemittance muon beams, no need for complicated compensation of non-linear effects, and a simpler combined-function magnet design with only dipole and quadrupole field components. The scheme utilizes only fixed magnetic fields, including those for injection and extraction.

We are currently studying the dynamic aperture and momentum acceptance of the arc. Earlier studies with a similar linear lattice yielded promising results [14]. We will investigate chromatic effects and, if necessary, implement their control and compensation. Another important aspect that requires investigation is the design sensitivity to magnet misalignments and magnetic field errors. Establishing tolerance levels on these errors is crucial for the costing of large aperture magnets.

\section{Summary and Closing Remarks}

The design study we have presented features a single pass linac is followed by a 4.5-pass, recirculating linear accelerator (RLA) in a "dogbone" configuration, exploring the elegance and flexibility of the RLA scheme.

The key advantage of a multi-pass RLA is its very efficient use of an expensive SRF linac, making the scheme elegant and flexible. Furthermore, the "dogbone" RLA is well suited for simultaneous acceleration of the $\mu^{+}$and $\mu^{-}$beams, while further compressing and shaping the longitudinal and transverse phase-space. Its inherently large momentum compaction, combined with off-crest acceleration in the subsequent pass through the linac, facilitates significant rotation in the longitudinal phase-space, which yields further compression of the longitudinal phase-space as the beam is accelerated. This feature makes it much superior to a straight linac. Finally, the "dogbone" scheme can be extended into a cascade of RLAs, as was envisioned within MASS [1]: the $5 \mathrm{GeV}$ RLA (NuMAX) was followed first by a larger RLA to $63 \mathrm{GeV}$ 
(Higgs Factory), and finally by another RLA to reach $\mathrm{TeV}$-scale energies (multi-TeV Muon Collider). That is why a multi-pass ( $>8$ ) "dogbone" RLA, configured with the proposed FFAGlike arcs, would be the technology of choice for the extreme muon acceleration needed for future muon facilities ( $\mathrm{TeV}$ scale and beyond).

\section{References}

[1] "Enabling Intensity and Energy Frontier Science with a Muon Accelerator Facility in the U.S.": White Paper Submitted to the 2013 U.S. Community Summer Study of the Division of Particles and Fields of the American Physical Society, August 2013.

[2] “International Design Study for the Neutrino Factory - Interim Design Report" arXiv:1112.2853v1 [hep-ex] 13 Dec 2011.

[3] S.A. Bogacz, "Beam Dynamics of Muon Acceleration for Neutrino Factory," Journal of Physics G: Nuclear and Particle Physics, 29, 1723, (2003).

[4] S.A. Bogacz, "Low Energy Stages - "Dogbone" Muon RLA," Nucl. Phys. B Proc. Suppl. 149, pp. 309-312 (2005).

[5] J.S. Berg, S.A. Bogacz, S. Caspi, J. Cobb, R. C. Fernow, J.C. Gallardo, S. Kahn, H. Kirk, D. Neuffer, R. Palmer, K. Paul, H. Witte, M. Zisman, "Cost-effective Design for a Neutrino Factory," Phys. Rev. ST-AB 9,011001(2006).

[6] S. Geer and M.S. Zisman, "Neutrino Factories: Realization and physics potential," Prog. in Part. and Nucl. Physics 59631 (2007).

[7] S.A. Bogacz, "Maximizing Number of Passes in Muon RLA," in Neutrino Factories, Superbeams and Betabeams: 9th International Workshop on Neutrino Factories, Superbeams, and Betabeams NuFact07, O. Yasuda, N. Mondal, and C. Ohmori, eds., vol. 981 of AIP Conference Proceedings, pp. 324-326, AIP, (2008).

[8] C. Ankenbrandt, S.A. Bogacz, A. Bross, S.Geer, C. Johnstone, D. Neuffer, R. Palmer, M. Popovic, "Low Energy Neutrino Factory Design," Phys. Rev. ST-AB 12, 070101 (2009).

[9] V. Lebedev, “Optim.” http://pbar.fnal.gov/organizationalchart/lebedev/OptiM/optim.htm

[10] G.M. Wang, S.A. Bogacz, R.P Johnson, D. Trbojevic, "Multipass Arc Lattice Design for Recirculating Linac Muon Accelerators," in Proc. PAC"09, Vancouver, BC, Canada.

[11] V.S. Morozov, S.A Bogacz, K.B. Beard, "Muon Acceleration with RLA and Non-scaling FFAG Arcs," in Proc. IPAC"10, Kyoto, Japan.

[12] V.S. Morozov et al., "Matched Optics of Muon RLA and Non-scaling FFAG arcs," in Proc. PAC"11, New York, NY, USA.

[13] D. Trbojevic et al., Phys. Rev. ST-AB 8, 050101 (2005).

[14] V.S. Morozov, S.A Bogacz, Y.R. Roblin, K.B. Beard, "Linear Fixed-field Multipass Arcs for Recirculating Linear Accelerators, PRST-AB,15, 060101 (2012). 